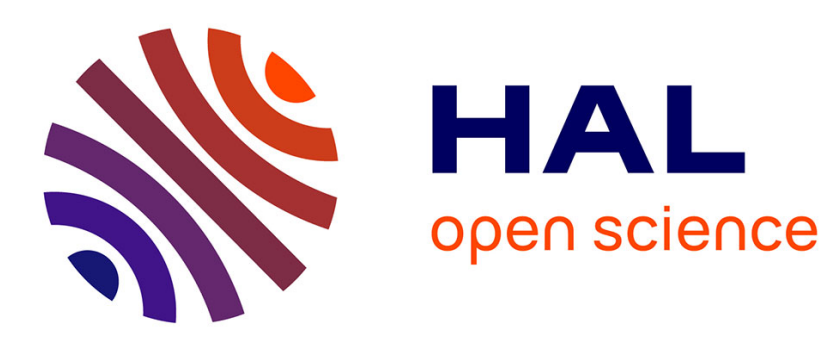

\title{
5G Internet of Radio Light Positioning System for Indoor Broadcasting Service
}

Lina Shi, Dayu Shi, Xun Zhang, Benjamin Meunier, Hequn Zhang, Zhan

Wang, Andrei Vladimirescu, Wei Li, Yue Zhang, John Cosmas, et al.

\section{To cite this version:}

Lina Shi, Dayu Shi, Xun Zhang, Benjamin Meunier, Hequn Zhang, et al.. 5G Internet of Radio Light Positioning System for Indoor Broadcasting Service. IEEE Transactions on Broadcasting, 2020, pp.1-11. 10.1109/TBC.2020.2981755 . hal-02559008

\section{HAL Id: hal-02559008 https://hal.science/hal-02559008}

Submitted on 30 Apr 2020

HAL is a multi-disciplinary open access archive for the deposit and dissemination of scientific research documents, whether they are published or not. The documents may come from teaching and research institutions in France or abroad, or from public or private research centers.
L'archive ouverte pluridisciplinaire HAL, est destinée au dépôt et à la diffusion de documents scientifiques de niveau recherche, publiés ou non, émanant des établissements d'enseignement et de recherche français ou étrangers, des laboratoires publics ou privés. 


\title{
5G Internet of Radio Light Positioning System for Indoor Broadcasting Service
}

\author{
Lina Shi ${ }^{(}$, Dayu Shi, Xun Zhang ${ }^{(}$, Benjamin Meunier, Hequn Zhang $\left.{ }^{(}\right)$, Zhan Wang, Andrei Vladimirescu, \\ Wei Li, Yue Zhang, John Cosmas, Kareem Ali, Nawar Jawad ${ }^{\circledR}$, Rudolf Zetik, Eric Legale, \\ Matteo Satta, Jintao Wang ${ }^{\mathbb{D}}$, and Jian Song
}

\begin{abstract}
Many features of 5G are definitely important to the broadcasting service, including diverse content services such as follow-me TV, video-on-demand, but also gaming, Virtual reality (VR) and Augmented reality (AR) and many others. Meanwhile, those services depend more and more on the user's position accuracy, especially in indoor environment. With the increase of broadcasting data traffic indoors, to obtain a highly accurate position is becoming a challenge because of the impact of radio interference. In order to support a high-quality indoor broadcasting service, a high-accuracy positioning, radiation-free, and high-capacity communication system is urgently needed. In this paper, a $5 \mathrm{G}$ indoor positioning system is proposed for museums. It utilizes unlicensed visible light of the electromagnetic spectrum to provide museum visitors with high-accuracy positioning, multiple forms of interaction services, and highresolution multimedia delivery on a mobile device. The geographic data and the location-related data integrated into the 5G New Radio (NR) waveform are detailed. A general-purpose system architecture is provided and some basic techniques to enhance system performance are also investigated. A prelimi-
\end{abstract}

Manuscript received December 1, 2019; revised March 2, 2020; accepted March 3, 2020. This work was supported in part by the Collaborative EUChina Project funded by the National Key Research and Development Program of China under Grant 2017YFE011230, and in part by the European Union's Horizon 2020 Research Program through the Internet of Radio-Light Project under Grant H2020-ICT 761992. Part of this paper have been published in the Proceedings of the 2018 25th IEEE International Conference on Electronics, Circuits and Systems (ICECS) (pp. 61-64), Bordeaux, France. (Corresponding author: Xun Zhang.)

Lina Shi, Dayu Shi, Xun Zhang, Zhan Wang, and Andrei Vladimirescu are with the Department of Electronic and Computer Engineering, Institute Supérieur d'Electronique de Paris, 75006 Paris, France (e-mail: lina.shi@isep.fr; xun.zhang@isep.fr).

Benjamin Meunier, John Cosmas, Kareem Ali, and Nawar Jawad are with the Department of Electronic and Computer Engineering, Brunel University, Uxbridge UB8 3PH, U.K. (e-mail: benjamin.meunier@brunel.ac.uk).

Hequn Zhang and Yue Zhang are with the Department of Engineering, University of Leicester, Leicester LE1 7RH, U.K. (e-mail: yue.zhang@leicester.ac.uk).

Wei Li is with the Department of Engineering, Viavi Solutions, Stevenage SG1 2AN, U.K. (e-mail: wei.li@ viavisolutions.com).

Rudolf Zetik is with the Department Wireless Distribution Systems, Fraunhofer Institute for Integrated Circuits IIS, Fraunhofer-Gesellschaft zur Foerderung der Angewandten Forschung E.V., 91058 Erlangen, Germany (e-mail: rudolf.zetik@iis.fraunhofer.de).

Eric Legale and Matteo Satta are with the Research and Development Department, Issy Média, 92130 Issy-Les-Moulineaux, France (e-mail: eric.legale@ville-issy.fr).

Jintao Wang and Jian Song are with the Department of Electronic Engineering, Research Institute of Information Technology, Tsinghua National Laboratory of Information Science and Technology, Tsinghua University, Beijing 100084, China (e-mail: wangjintao@tsinghua.edu.cn).

Color versions of one or more of the figures in this article are available online at http://ieeexplore.iee.org.

Digital Object Identifier 10.1109/TBC.2020.2981755 nary demonstration is built in the laboratory environment. It supports over 45.25 Mbps data rate and a mean positioning error of $0.18 \mathrm{~m}$.

Index Terms-5G new radio (5G NR), visible light communication (VLC), location-based services (LBS), indoor broadband broadcasting system, 5G Internet of radio light (5G IoRL).

\section{INTRODUCTION}

$\mathbf{I}$ $\mathrm{N}$ RECENT years, with the emergence of $5 \mathrm{G}$ technologies, the convergence of $5 \mathrm{G}$ with broadcasting services may have a much more transformative impact than expected [1]-[4]. The $5 \mathrm{G}$ forum white paper [5] pointed clearly that the next $\left(5^{\text {th }}\right)$ generation wireless network provides a safer, more secure, customizable and intelligent building network that reliably delivers increased throughput (greater than 10Gbps) from access points pervasively located within buildings, whilst minimizing interference and electromagnetic field exposure and providing location accuracy from $10 \mathrm{~m}$ to less than $1 \mathrm{~m}$ for $80 \%$ of occasions, and better than $1 \mathrm{~m}$ for indoors. With those features, 5G will be essential for a myriad of new broadcasting services based on accurate location awareness and other contextual information [6].

Meanwhile, the demand for mobile data broadcasting and location-related broadcasting service in broadband networks is rapidly increasing. According to the data from Cisco, it predicts that broadcasting data traffic will reach approximately 77 Exabytes per month by 2020, where $70 \%$ to $90 \%$ of the overall data traffic will occur in indoor environments [7]. Apart from enjoying high-speed data broadcasting services like 4K live broadcasting, VR, AR, High Dynamic Range (HDR) and high-definition (HD) video surveillance, people also put much higher requirements for related location-based services (LBS) [8]. According to the report in [9], there is a minimum of $52.63 \%$ of frequently used devices with positioning capabilities, such as mobile phones, vehicle satellite navigation, fitness devices, iWatch, iPod, iPad. These devices with positioning capabilities facilitated the growth of the location-related broadcasting services.

However, the accurate geographic data broadcasting is not only relying on the characteristics of the up-link and down-link signals but also on new technologies and network configurations. For example, Global Navigation Satellite Systems (GNSS) (e.g., BeiDou, Galileo, GLONASS, and GPS), Terrestrial Beacon Systems (TBS), Bluetooth, WLAN, 
sensors etc. [9]. Although these non-3GPP technologies have been widely used thanks to their low-cost, they cannot guarantee to provide satisfactory positioning resolution for $5 \mathrm{G}$ network especially in the indoor environment where it is lack of service coverage and poor accuracy.

In this context, this paper proposes an integrated solution of indoor positioning systems and broadcasting service based on Internet of Radio Light (IoRL) infrastructure [10]. Visible Light Communication (VLC) system and VLC-based positioning method are adopted to fulfil the requirement in terms of capacity and positioning accuracy with low-cost LED devices. The proposed system is evaluated in a museum scenario and will be deployed in the museum "Musée Nationale de la Carte à Jouer" of Issy-les-moulineaux in Paris in June 2020.

The main contributions of this paper can be summarized as follows:

1) The unified $5 G$ NR signal is designed as the positioning signal over VLC, the geographic data and the locationrelated data is in a unified $5 \mathrm{G}$ NR frame structure and some special adaptations for the VLC case are studied. Furthermore, the positioning procedure is also proposed for the first time.

2) A real-time demonstration which has a data rate of around $45.25 \mathrm{Mbps}$ within a bandwidth of $10 \mathrm{MHz}$ by using 3.6 W LED lamp is implemented in laboratory. The system supports an Error Vector Magnitude (EVM) less than $4 \%$ with different modulation scheme (QPSK, 16-QAM, 64-QAM and 256-QAM). In view of positioning performance, to the best of our knowledge, our work is the first to demonstrate that by using $5 \mathrm{G}$ NR signal, VLC-based indoor positioning system can achieve a mean positioning error of $0.18 \mathrm{~m}$.

The rest of this paper is organized as follows: Section II highlights the related positioning technologies for broadcasting services. Section III describes the location-based broadcasting applications for museum scenarios. The proposed system architecture diagram together with the main components are introduced in Section IV. Section V provides the definition of the geographic data and the location-related data; the positioning procedure is also investigated. The proposed end user applications for museums are introduced in Section VI. The details of the demonstration setup and evaluation are presented in Section VII. The system features and capabilities are illustrated in Section VIII. Section IX highlights the conclusions.

\section{Positioning TeChNOlogies FOR BROADCASTING SERVICES}

This section reviews the various indoor positioning technologies for broadcasting services in recent years and their drawbacks.

The Global Navigation Satellite Systems (GNSS), as the most widely used technology in the current 4G long term evolution (LTE) standards, enables determining the outdoor geographic location within few meters of accuracy and thus supports lots of location-based broadcasting applications and services [11] such as navigation, tracking and Location-Based Social Networking. However, the GNSS cannot provide users with the same level of positioning accuracy, continuity, and reliability in the indoor environment due to the attenuation and reflection by buildings. To cope with this issue, the research of emerging technologies for indoor positioning is on-going [12]-[22], for example, Wi-Fi [18], Frequency Modulation (FM) broadcast technology (ex. digital television terrestrial broadcasting (DTTB) [19]), Bluetooth [16], [20], RFID [21], Ultra-wideband (UWB) [13]-[17], ZigBee [14], etc. The advantages and disadvantages of these technologies are compared in detail in [22]. Besides these well-known positioning technologies, $5 \mathrm{G}$ proposes a number of promising positioning technologies, which can be applied at mmWave frequencies and which is a feasible solution in 5G era with two advantages: 1) large available bandwidth to meet the increasing demand for mobile data broadcasting; 2) the possibility to pack a large number of antenna elements in smartphone or others mobile device. A large number of antenna elements enables massive MIMO and high accurate beamforming [23]. This is helpful for 2D/3D position and $3 \mathrm{D}$ orientation [24]. Although the mmWave-based positioning technology can meet the positioning accuracy requirements for the $5 \mathrm{G} \mathrm{LBS}$, the high time resolution required from the mmWave-based positioning system needs a high synchronization clocking source with extreme low phase noise and the cost of mmWave frontend is still considerably high. Compared with these Radio-Frequency (RF) solutions mentioned above, the advantages of visible light technology include achieving high positioning accuracies, high transmission capacity, interference-free while maintaining a low deployment cost [25], [26], [28], [29]. Apart from that, VLCbased positioning system can be a reasonable alternative to those RF-based positioning systems in some RF prohibited areas such as hospitals, aircraft cabins and oil plants [30].

In general, the current VLC applications are very rich and broad, with opportunities in the most diverse areas. High speed transmission and high accuracy indoor positioning are the most widely discussed among these areas. Orthogonal frequencydivision multiplexing (OFDM) is most extensively used in VLC systems to achieve high-speed transmission, because of its high spectral efficiency and low-complexity implementation. Gigabit transmissions using OFDM-based VLC have recently been achieved and reported [31], [32]. For example, $10 \mathrm{~Gb} / \mathrm{s}$ is achieved by using a GaN violet micro-LED [31]. Apart from OFDM, multiple channel multiplexing techniques such as wavelength division multiplexing (WDM) has been used to realize high speed data rate goal. Reference [33], [34] reported WDM with the use of RGB laser diodes (LD) demonstrating the rate of $8 \mathrm{~Gb} / \mathrm{s}$ and $14 \mathrm{~Gb} / \mathrm{s}$, respectively. However most importantly from the positioning perspective, visible light-based systems have the ability to estimate an object's position at the accuracy within the centimeter range [36]. For example, according the summary about the state-of-theart of indoor VLP in [37], the highest positioning accuracy is proposed by [38], in which a positioning accuracy of $0.69 \mathrm{~cm}$ is achieved in an indoor environment of $4 \mathrm{~m} \times 4 \mathrm{~m}$ $\mathrm{x} 6 \mathrm{~m}$ by using differential evolution algorithm. These attractive performance data illustrate that visible light technology is a promising candidate for serving the high data rate as 


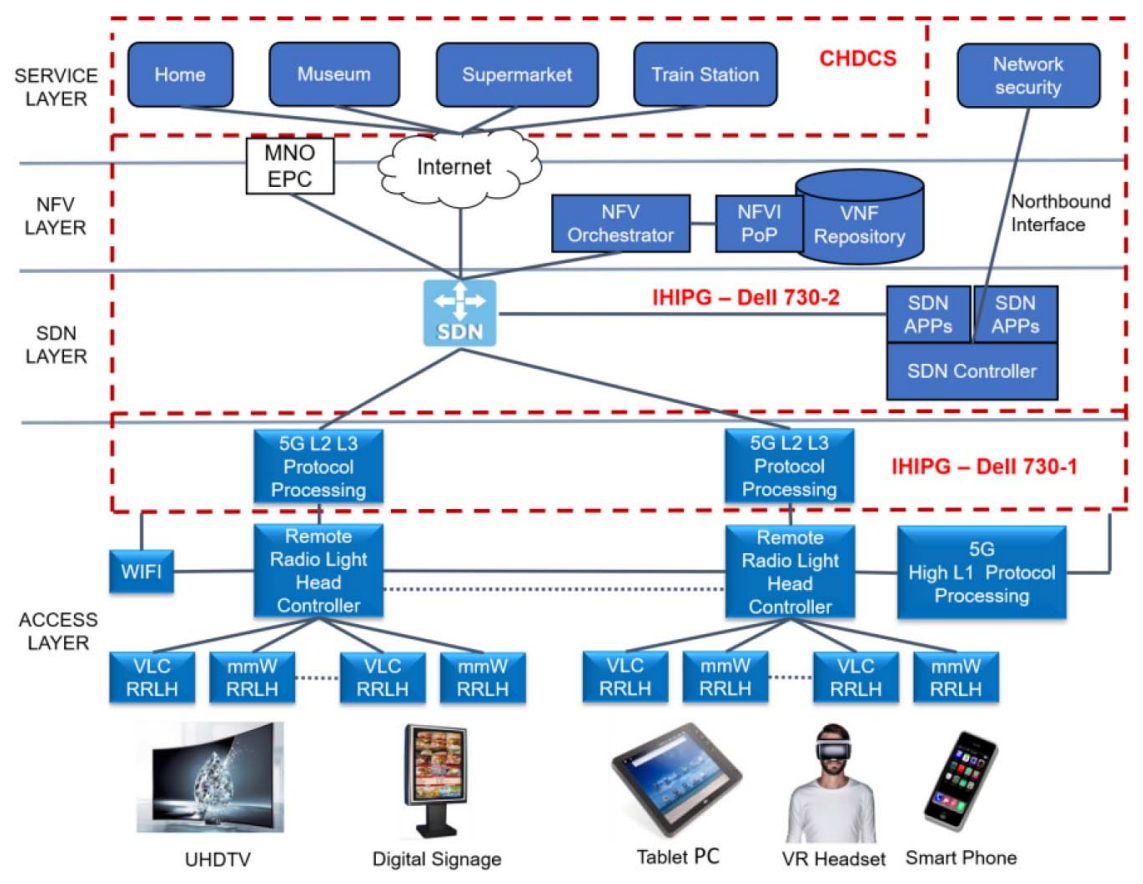

Fig. 1. Internet of Radio Light infrastructure.

well as positioning accuracy demands of $5 \mathrm{G}$ communication systems.

Our proposition is based on the IoRL framework, which is a typical indoor solution that integrates VLCbased positioning system into 5G network with Intelligent Home IP Gateway (IHIPGW) and Software Defined Networks (SDN)/Network Function Virtualization (NFV) network paradigm and which provides Virtual Network Functions (VNFs). It is structured in four layers including the service layer, NFV layer, SDN layer, and the access layer. The system architecture is shown in Fig. 1. IoRL is also compatible with existing system/standards, e.g., IEEE 802.11, 802.15, 3GPP. There are two main benefits namely delivering a total of $10 \mathrm{Gbs}$ data rates in a building with a latency under $1 \mathrm{~ms}$ for future $5 \mathrm{G}$ broadcasting service; providing location sensing services with sub $10 \mathrm{~cm}$ accuracy for upcoming indoor $5 \mathrm{G}$ era. This is made possible by modulating a high-powered communication LED within the existing lighting infrastructure for VLC. Furthermore, we investigated the reuse of the same Transport Blocks of 5G NR and adding some improvements/adaptation for the VLC case. This may simplify and reduce prices of the terminals and the access points. All the above-mentioned capabilities have brought great motivation for VLC research in 5G networks.

The intention of the proposed system is to employ a location-based broadcasting VNF based on IoRL framework into museum scenarios to provide an improved Quality of Experience (QoE) and a high accuracy LBS by using low-cost VLC-based positioning technology.

\section{LOCATION-BASED BROADCASTING APPLICATIONS FOR MUSEUM SCENARIOS}

The museum as a typical scenario for indoor broadband and broadcasting systems, which has attracted more and more attention in recent years. It requires to display position and direction on a map of the mobile device and broadcasts also the corresponding information about its surroundings, closeby objects, or time to destination. There are three typical requirements for the geographic data broadcasting.

\section{A. Indoor Location-Based Data Access}

Research in [39] applies iBeacon technology in museums to provide visitors with indoor location-based data access. With the aid of the geographic data information, staff members of museums can provide the exhibit's information to immediately inform the museum visitors. However, this system can only provide the rough estimation of the position (between 3 and 5 meters).

\section{B. Indoor Location Monitoring and Guiding}

Research in [40] investigated to implement a museum monitoring and guiding application using Bluetooth Low Energy (BLE) beacon.

The visitor's position is calculated by BLE beacon signal strength (RSS) at the location server. The server then broadcasts the information about the artifact near the visitor's location through the Internet. Even as the location accuracy has been improved in [40] compared to [39], visitors need additional connected readers to obtain higher location accuracy (around 2 meters). This method is not convenient for visitors.

Another example is the "Smart Museum" system proposed in [41], where Near-field communication (NFC) and RadioFrequency Identification (RFID) technologies are used for indoor localization. However, this solution is required to install RFID readers on mobile devices, which are expensive and not convenient. 


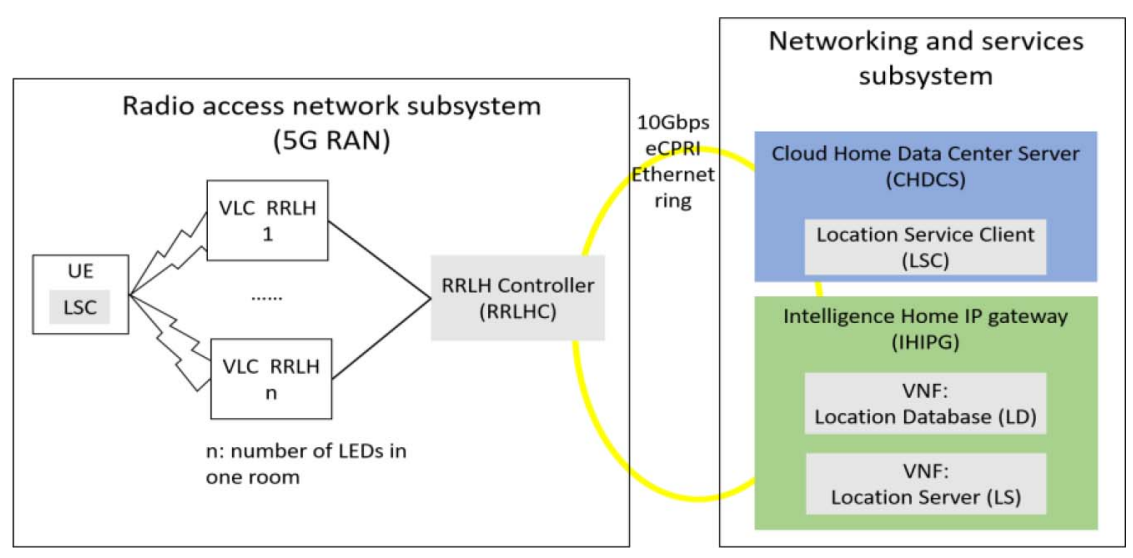

Fig. 2. 5G IoRL positioning system architecture diagram.

\section{Interaction Between Visitors and Systems}

Research in [42] presented a simulation framework for a location-based VR museum, which is a RF-based location system operating in the Gigahertz band. However, there is no practical demonstration based on their simulation.

The limitation of the aforementioned works is that they cannot provide satisfactory positioning accuracies required for future broadcasting service. To deal with the limitation and meet all requirements above, a high accuracy $5 \mathrm{G}$ positioning system using VLC is presented in the next section.

\section{System ARChitecture Diagram}

This section details the 5G IoRL positioning system architecture presented in Fig. 2. The architecture consists of two subsystems: the networking and services subsystem and the radio access network subsystem (5G RAN). Both parts are connected together through a 10Gbps enhanced Common Public Radio Interface (eCPRI) Ethernet ring.

The networking and services subsystem consist of the Cloud Home Data Center Server (CHDCS) and IHIPGW, which incorporate with the NFV technologies offering a flexible infrastructure.

The 5G RAN subsystem consists of the Remote Radio Light Head Controllers (RRLHC), the VLC RRLHs (LEDs) and UE. The number of RRLHC $(N)$ depends on the number of rooms in building. The number of VLC RRLH $(n)$ depends on the number of LEDs required in one room. There can be any number of UEs in a room.

In summary, there are four main components relevant to location in these two subsystems: the location server (LS), the location database (LD) in the IHIPG, the RRLH controller (RRLHC) in the RAN and the location service client (LSC) in the UE.

- The LS is a VNF implemented within the IHIPGW to estimate the coordinates of UE;

- The LD is another VNF implemented within the IHIPGW. There are three different sets of parameters stored in the LD:

1) Received signal strength (RSS) of the geographic data obtained by UE within geographic coverage area of a room;
2) Estimated coordinates of UE;

3) Coordinates of all LEDs and floor plans.

- RRLHC is used to transmit geographic data with known power, where one RRLHC is responsible for signal dissemination within one room;

- The LSC is a software application (e.g., indoor location monitoring and guiding application) inside both the $\mathrm{UE}$ and the CHDCS. It provides position information for monitoring, guiding and interactive applications (ex. VR gaming application) in the form of RSS measurements.

\section{Geographic Data, Location-Related Data, and Positioning Procedure in Museum}

\section{A. The Geographic Data and the Location-Related}

Data Integration in $5 G$ NR Frame

Each geographic data represents one LED's ID. This ID is generated by 5G RAN and broadcasted through VLC link.

The geographic data and location-related data are integrated in 5G NR frame, which is illustrated in Fig. 3. They are allocated in the Physical Downlink Shared Channel (PDSCH) slots. In each slot, the $3^{\text {rd }}$ to $10^{\text {th }}$ OFDM symbols are used to transmit the location-related data such as the multimedia contents about the close-by exhibitions. The last four OFDM symbols transmit four geographic data, where only one particular subcarrier frequency $f$ is used to carry the respective geographic data. Since one time slot duration is $0.5 \mathrm{~ms}$, each data is obtained by UE every 36 us $(0.5 \mathrm{~ms} / 14)$ at least.

In the next subsection, the indoor positioning procedure will be presented.

\section{B. Indoor Positioning Procedure}

The indoor positioning procedure involves interaction between RRLHC, LD, LS, LSC and the UE, which is illustrated in Fig. 4.

1) Measurement of Location Relevant Parameters (See the Red Lines in Fig. 4):

a. The UE sends the positioning request to the RRLHC;

b. The RRLHC sequentially broadcasts a series of LEDs' IDs at Time Division Multiplex (TDM) mode;

c. The UE receives the IDs from all LEDs in one room and reports them to the LD by using TCP/IP packages. 


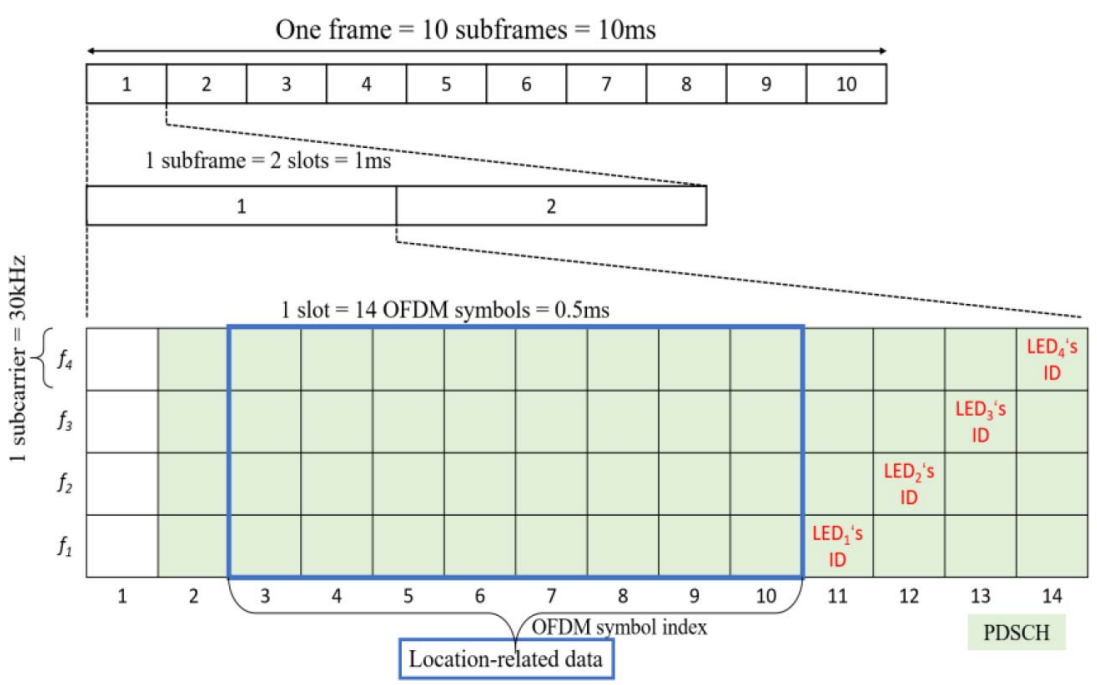

Fig. 3. Definition of the geographic data and the location-related data in 5G NR frame. One frame consists of 10 subframes, each subframe has two slots and each slot has 14 OFDM symbols. In the frequency domain, the subcarrier spacing is $30 \mathrm{kHz}$.

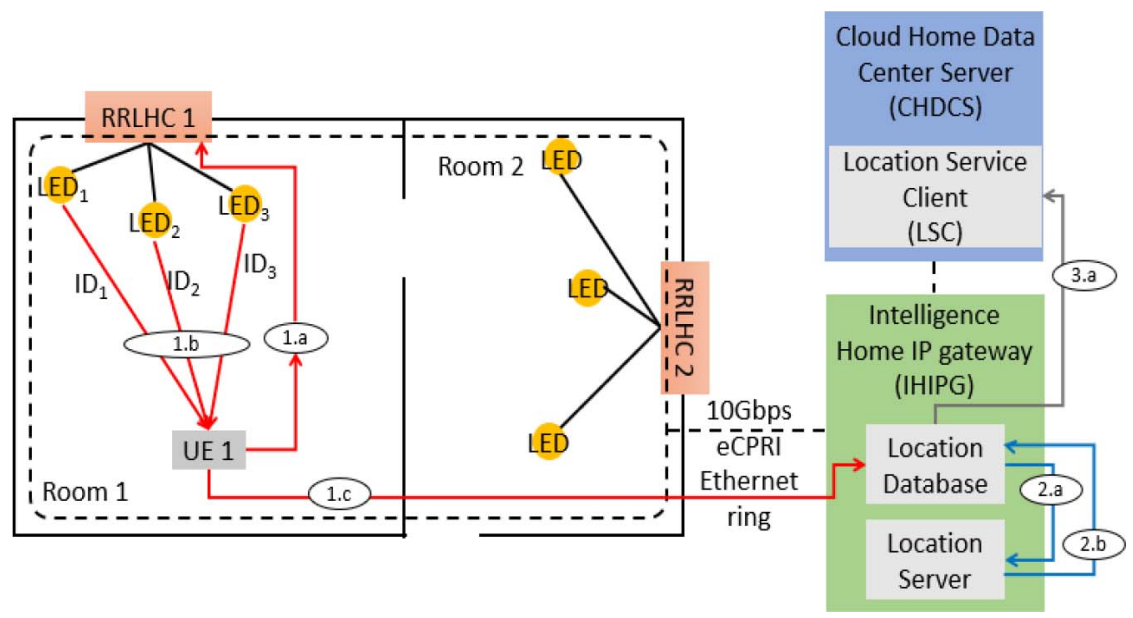

Fig. 4. Geographic data broadcasting and positioning procedure.

2) Position Estimation (See Blue Lines):

a. The LS retrieves the latest IDs data and coordinates of corresponding LED from the LD to perform the UE's position estimation;

b. The LS writes the UE's position estimation results to the LD.

3) Exploitation of Position Estimation:

a. The LSC provides location-related broadcasting service to user by retrieving the UE's position estimation from the LD.

\section{VLC Positioning Algorithm}

Common positioning algorithms used for VLC indoor localization systems are based on the triangulation method, and they need to estimate the distance or angle between transmitter and receiver. There are many ways to achieve the distance or angle such as received signal strength (RSS) [25], [43], time of arrival (TOA) [14], [27], time difference of arrival (TDOA) [30], and angle of arrival (AOA) [44].
AOA positioning algorithm can achieve high accuracy, but it needs to deploy a sensor array, which is very expensive for users. On the other hand, the TOA or TDOA method is a positioning algorithm based on transmission time. However, TOA requires the transmitted signals to be very accurately synchronized. The need for accurate transmitter synchronization can be avoided if TDOA [45] rather than TOA is used. However, the short distance between transmitter and the receiver results in short transmission time in indoors. Thus, extremely accurate time measurement is required. On the contrary, RSS does not need high accuracy synchronization and high frequency clocking compared to the TOA and TDOA methods. Furthermore, VLC RSS parameters are easy to obtain using a single photodetector without the need for auxiliary devices. To take the cost, complexity and accuracy of indoor positioning into consideration, RSS based positioning is preferred due to its low cost, low complexity and high accuracy [25].

Therefore, this section elaborates the RSS positioning algorithm in our proposition. Based on RSS triangulation 


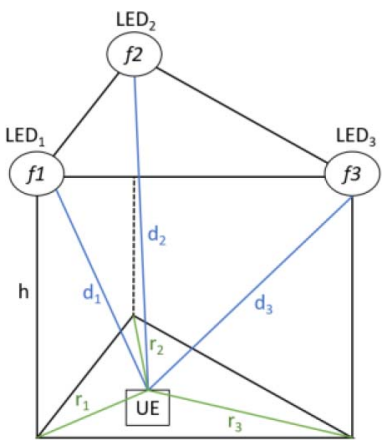

Fig. 5. Positioning algorithm diagram.

positioning algorithm, minimum three LED's ID signal power should be registered for estimating UE's position. In the example shown in Fig. 5, three LED's ID signal $f 1, f 2$ and $f 3$ are the dedicated subcarrier frequencies with the three highest received power. Therefore, the distance $d$ between the LED and UE can be calculated by using equation (1)

$$
d=\sqrt[(m+3)]{\left(\frac{(m+1) A_{r} h^{(m+1)}}{2 \pi}\right) \frac{P_{T}}{P_{R}}}
$$

where $A_{r}$ is the effective area of the receiving surface of the UE. $h$ is a known constant about the vertical distance between the UE and the LED. $m$ is the order of Lambertian emission, which is relative to the semi-angle at half power of the LED denoted as $\varphi_{1 / 2}$.

$$
m=\frac{-\ln 2}{\ln \left(\cos \left(\varphi_{1 / 2}\right)\right)}
$$

The received power $P_{R}$ of the UE can be represented as:

$$
P_{R}=H(0) * P_{T}
$$

where $P_{T}$ is the transmitted light power of LED. $H(0)$ is VLC Line of Sight (LOS) channel gain between the LED and UE which can be shown as:

$$
H(0)=\frac{(m+1) A_{r} \cos ^{m}(\varphi) \cos ^{m}(\theta)}{2 \pi d^{2}}
$$

where $\varphi$ is the radiation angle between the LED and the UE, and $\theta$ is the angle of light incident to the receiving surface of the UE.

Thus, the distance $d_{1}, d_{2}$ and $d_{3}$ can be obtained respectively.

The projection distance $r_{1}, r_{2}$, and $r_{3}$ between LED and UE is expressed as shown below:

$$
r=\sqrt{d^{2}-h^{2}}
$$

The estimated coordinates of UE: $X_{e}\left(x_{e}, y_{e}\right)$ thus can be calculated as follows:

$$
\left\{\begin{array}{l}
\left(x_{e}-x_{1}\right)^{2}+\left(y_{e}-y_{1}\right)^{2}=r_{1}^{2} \\
\left(x_{e}-x_{2}\right)^{2}+\left(y_{e}-y_{2}\right)^{2}=r_{2}^{2} \\
\left(x_{e}-x_{3}\right)^{2}+\left(y_{e}-y_{3}\right)^{2}=r_{3}^{2}
\end{array}\right.
$$

where, $\left(x_{1}, y_{1}\right),\left(x_{2}, y_{2}\right)$ and $\left(x_{3}, y_{3}\right)$ are the known coordinates of three LED respectively. Equation (6) can be formed in a matrix format as (7)

$$
\boldsymbol{B} X_{e}=\boldsymbol{C}
$$

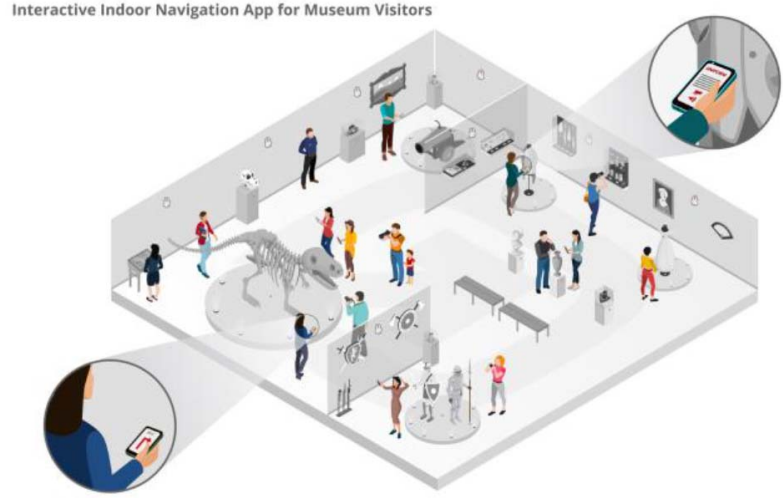

Fig. 6. Location-Based Data Access Application.

where $\boldsymbol{B}, \boldsymbol{C}$ and $X_{e}$ are defined as

$$
\begin{aligned}
\boldsymbol{B} & =\left[\begin{array}{ll}
x_{2}-x_{1} & y_{2}-y_{1} \\
x_{3}-x_{1} & y_{3}-y_{1}
\end{array}\right], X_{e}=\left[\begin{array}{l}
x_{e} \\
y_{e}
\end{array}\right] \\
\boldsymbol{C} & =\left[\frac{\frac{d_{1}^{2}-d_{2}^{2}+x_{2}^{2}+y_{2}^{2}-x_{1}^{2}-y_{1}^{2}}{d_{1}^{2}-d_{3}^{2}+x_{3}^{2}+y_{3}^{2}-x_{1}^{2}-y_{1}^{2}}}{2}\right]
\end{aligned}
$$

The estimated $X_{e}$ can then be obtained by the linear least squares.

$$
\widehat{X_{e}}=\left(\boldsymbol{B}^{T} \boldsymbol{B}\right)^{-1} \boldsymbol{B}^{T} C .
$$

\section{END User APPLICATIONS}

To demonstrate the performance requirements for broadcasting the geographic data mentioned in Section III, three different applications are developed as following.

\section{A. Location-Based Data Access Application}

As shown in Fig. 6, it allows the museum to deliver the specific description of the demonstrated objects to the visitors nearby yet within exhibition room. The application is able to view exhibits from the location database. The location database has been designed using Django architecture. The application requires the use of a database and an interface that will interact with the VLC access points and the database. Essentially the VLC access points would from now on lookup the data from the database, and the application developer would be able to access and edit the information in this database (see Fig. 7), making it easier to manage and control the application.

\section{B. Monitoring \& Guiding Application}

Monitoring application see Fig. 8, allows the museum to record the behavior of visitors listed below for further analysis.

- The visitor's path through the museum;

- The amount of time individual visitor spent at each exhibit;

- The most visited exhibit;

- The population density of visitors in the museum at any time.

According to the definition of LBS, LBS systems use realtime geo-data from a mobile device or smartphone to provide 


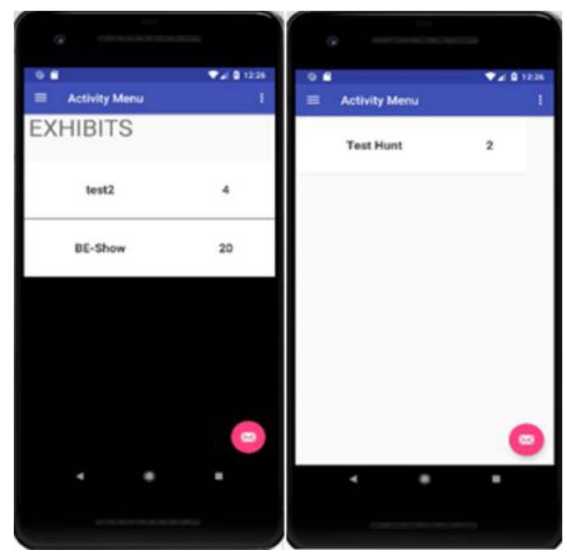

Fig. 7. Application fetching data from the location database.

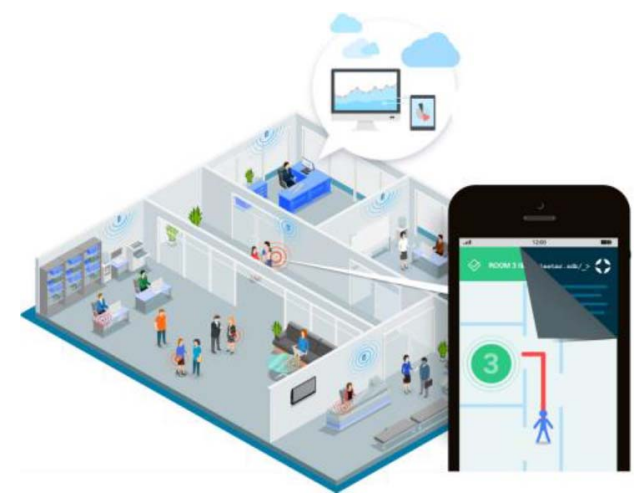

Fig. 8. Monitoring Application.

information, entertainment or security and to improve user experience. For the museum scenarios, our solution allows the visitors to access to a floor plan of the museum to help the visitor to navigate around it, as shown in Fig. 9.

As visitors move around the museum from exhibit to exhibit, the location of each visitor is estimated by RSS measurements and constantly updated on the user application database. The monitoring \& guiding application then accesses the user application database and retrieves appropriate LED's ID that covers that visitor. The downlink packets destined for specific visitor are then broadcasted by his serving LED and received by the corresponding visitor. Moreover, at the same time, a database records their behaviors, namely: the time each visitor spends at each exhibit, their path through the museum, the density of visitors in the museum at any one time etc. for analysis by the museum management and for increasing the user experience.

\section{Interaction Application}

Interaction can enhance the visitor user experience where the museums provide their exhibitions with massive bespoke digital information only through the individual visitors' request including VR or AR applications [46].

Once the received geographic raw data in the LD has been processed by the positioning algorithm in the LS to determine the UE's geographic position, it can then be used by user applications. The VR application can now retrieve this data to

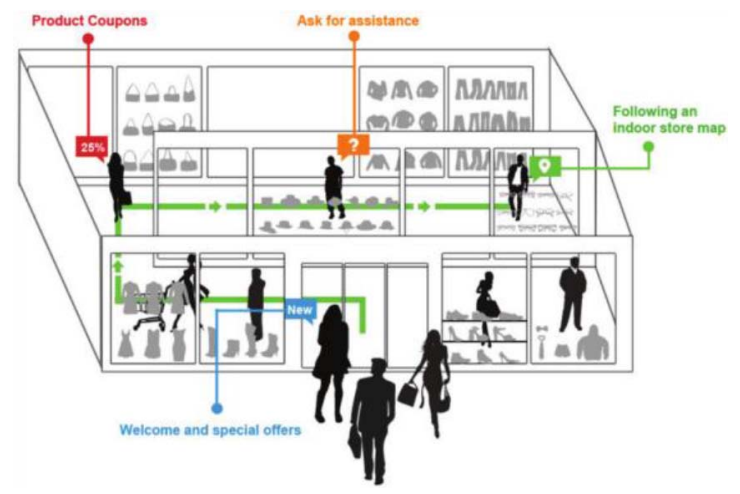

Fig. 9. Guiding Application.

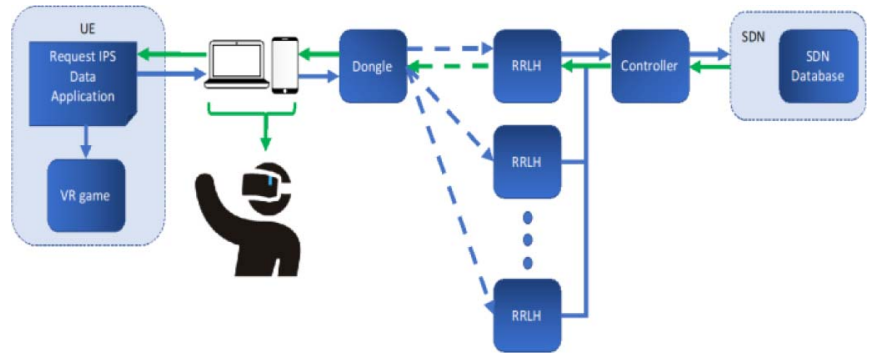

Fig. 10. VR gaming application.

TABLE I

THE LABORATORY ENVIRONMENT SETUP

\begin{tabular}{ll}
\hline \hline \multicolumn{1}{c}{ Parameter } & \multicolumn{1}{c}{ Value setting } \\
\hline Positioning Area $\left(\mathrm{L}^{*} \mathrm{~W}^{*} \mathrm{H}\right) / \mathrm{m}$ & $2.0 \times 1.0 \times 2.4$ \\
& $(-0.5,0.25,2.4), \quad 0.5,0.25$, \\
Positions of the four LEDs /m & $2.4)$, \\
& $(-0.5,0.6,2.4),(0.5,0.6,2.4)$ \\
Height of the PD / m & 0.175 \\
Effective area of PD /cm2 & $7.07 \mathrm{e}^{-2}$ \\
\hline \hline
\end{tabular}

determine the user's physical movements and thus their position in the virtual environment. The process of the position data dissemination is illustrated in the green line of Fig. 10. The procedure involves the mobile application sending out a request to the LD on the IHIPGW via the RRLHC. The location data is then outputted from the LD back to the application.

\section{The Demonstration And EVAluation}

\section{A. Demonstration Setup}

The demonstration is performed in a practical indoor environment and its feasibility has been shown through experiments, as shown in Fig. 11. This system is quite simple in composition and does not require any additional installation.

There are four commercial LEDs installed on the ceiling to broadcast the geographic data. Each LED is connected to a VLC modulator. A Silicon avalanche photodetector (Hamamatsu APD C5331-11) is used as the VLC receiver [47]. Related parameters of LED and of APD can be found in Table I. 

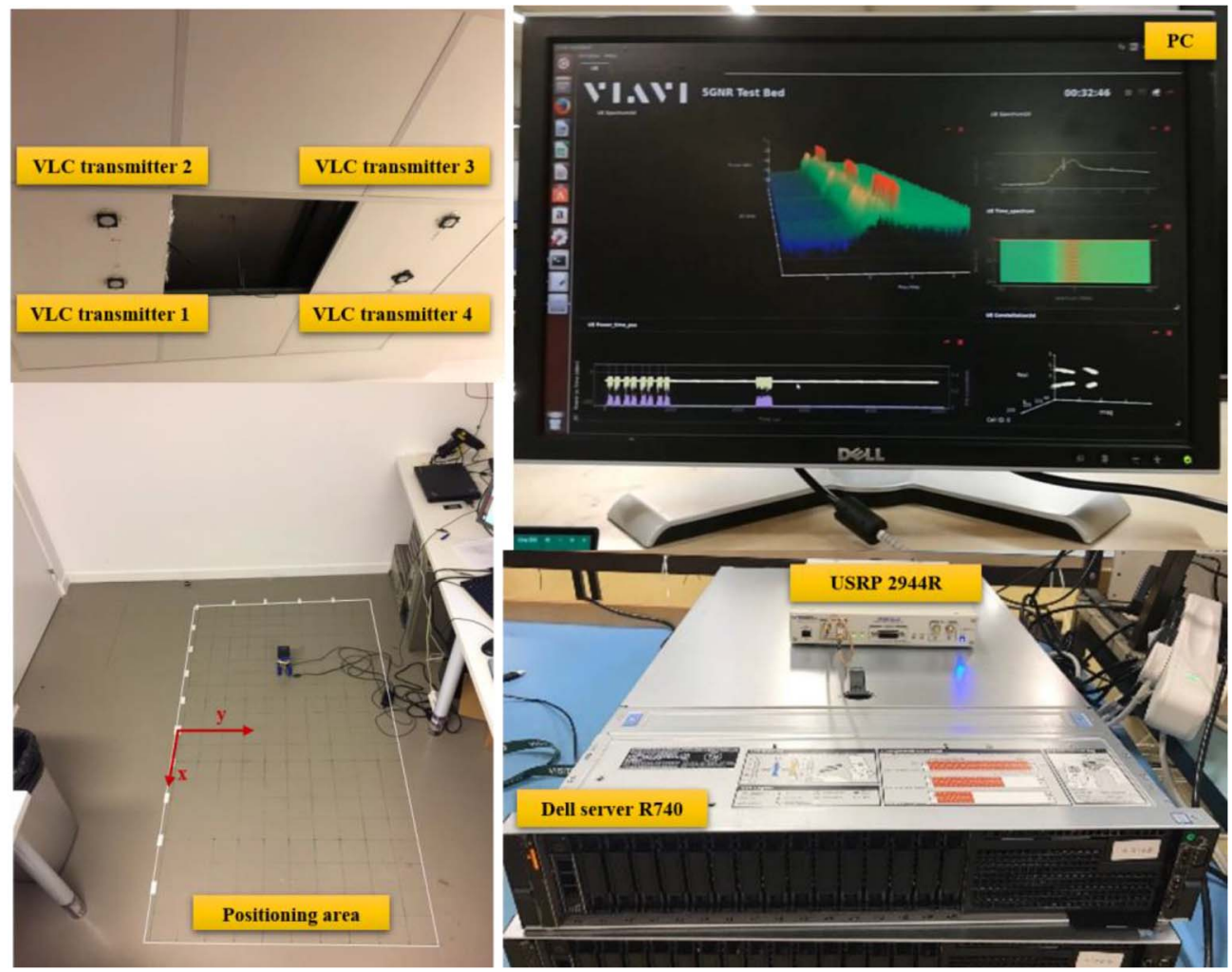

Fig. 11. The demonstration of the 5G Internet of Radio Light positioning system in the laboratory environment.

At the Tx, one Dell server R740 acts as the 5G NR transmitter and generates $5 \mathrm{G}$ baseband signals. The user data is modulated with different modulation schemes that include QPSK, 16-QAM, 64-QAM and 256-QAM. The modulated symbols are then assigned to the PDSCH of the 5G NR frame. After that, inverse Fast Fourier transform (IFFT) processing is applied to convert the OFDM frame to the time-domain. In addition, the 5G NR baseband signal is complex-valued data, to guarantee that the time-domain signal is real-valued in the IM/DD VLC system, IQ modulation is adopted. Subsequently, the time-domain discrete signal is scaled and passed through a digital-analog (D/A) converter by using USRP 2944R. Then, a DC bias is added to ensure the signal is in the quasilinear range of LED. At the end, the signal can be transmitted over VLC.

At the receiver side, the VLC signal is received by APD and digitized by analog-digital (A/D) converter of USRP 2944R. After receiving data from USRP, a UE Layer 1 processing software is used to do the Fast Fourier transform (FFT) and OFDM demodulation to recover the data from timedomain to frequency-domain. It estimates the channel response with pilot signal in PDSCH and uses zero forcing equalizer to recover the symbols. After that, it calculates the EVM and positioning error.

\section{B. Evaluation of the Demo}

1) Communication Performance: The demonstration can work well with white LED lamp in a normal laboratory indoor lighting environment. The system can provide a data rate of around $45.25 \mathrm{Mbps}$ with a bandwidth of $10 \mathrm{MHz}$ from $10 \mathrm{MHz}$ to $20 \mathrm{MHz}$.

The constellation diagrams of the received signal as well as the signal's Error Vector Magnitude (EVM) are measured for QPSK, 16-QAM, 64-QAM and 256-QAM modulation schemes, as shown in Fig. 12. The system supports an EVM less than $4 \%$ with those kinds of schemes and their constellation points are concentrated, which means that our system has a good communication link quality and ensures the probability of successful demodulation. In addition, QPSK, 16QAM and 64QAM can meet the 3GPP EVM recommendation: EVM $_{\mathrm{QPSK}}<17.5 \%$, $\mathrm{EVM}_{16-\mathrm{QAM}}<12.5 \%$, and $\mathrm{EVM}_{64-\mathrm{QAM}}<8 \%$ which are illustrated in Table 6.5.2-1 in [48]. The EVM of the 256-QAM and $3 \mathrm{GPP}$ recommendation in this modulation scheme are also extremely close.

2) Positioning Accuracy: Four single-tone signals are used to represent the geographic data of four LEDs' ID: $300 \mathrm{kHz}$, $600 \mathrm{kHz}, 900 \mathrm{kHz}$ and $1200 \mathrm{kHz}$ with $-3 \mathrm{dBm}$ transmitter power. The positioning accuracy is evaluated by the PE defined in equation (11) as follows:

$$
P E=\sqrt{\left(x_{e}-x_{r}\right)^{2}+\left(y_{e}-y_{r}\right)^{2}}
$$

where $x_{r}, y_{r}$ and $x_{e}, y_{e}$ are the coordinates of the reference points and estimated points, respectively.

We plot the distribution of reference points and estimated points in Fig. 13 (a). Under the experimental environment, 6*11 uniform distributed reference points are selected over the positioning area. Each reference point is measured 5 times thus there are $330(6 * 11 * 5)$ estimated points in total. 

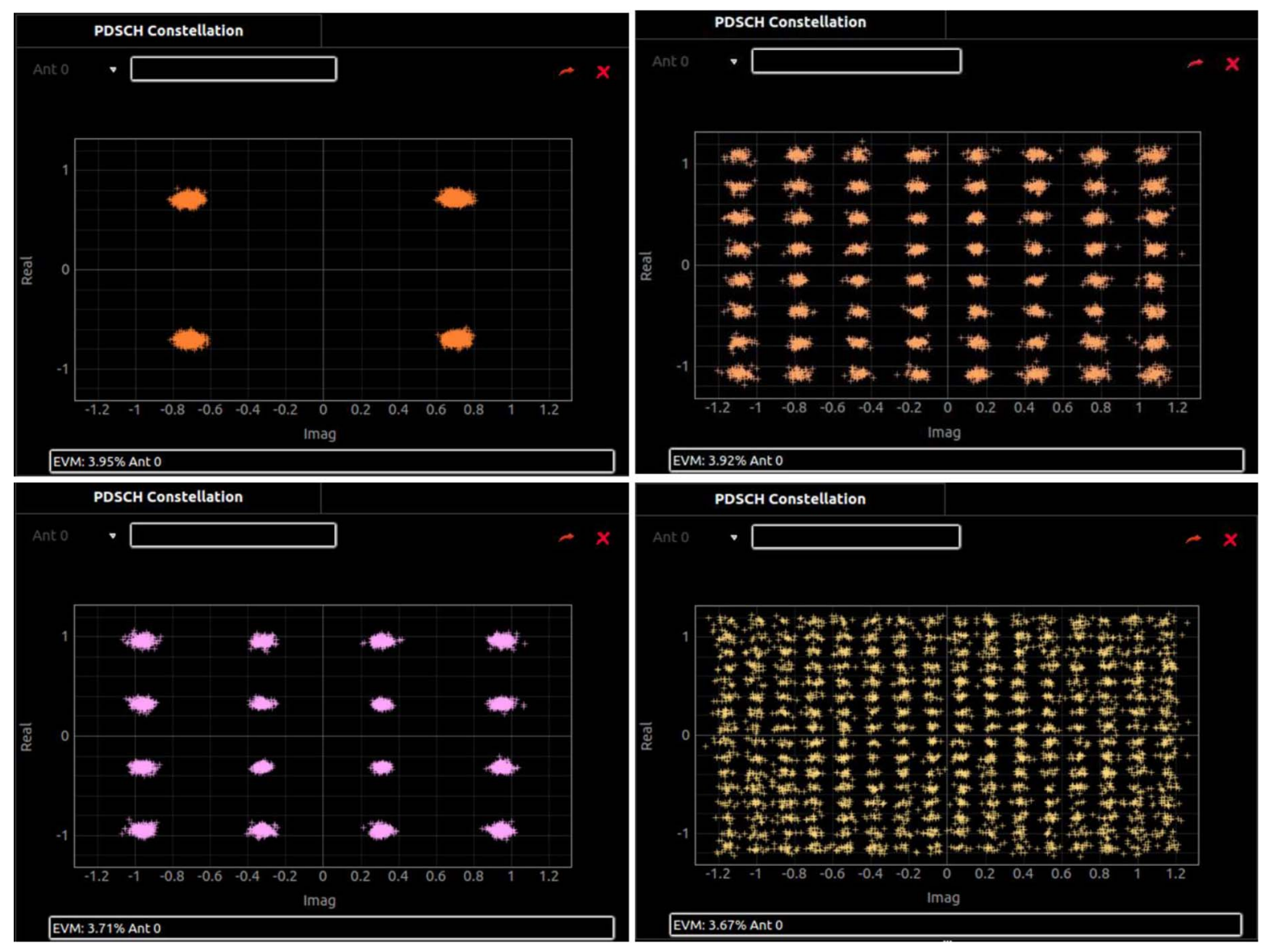

Fig. 12. The measured constellation diagrams of the received signal for proposed system (left upper corner: QPSK, left lower corner: 16-QAM, right upper corner: 64-QAM, right lower corner: 256-QAM).

The distribution of PEs is illustrated in Fig. 13 (b). Although there are significant PEs especially in the room corners, it obtains a good positioning performance in the room center. This can be explained by its sensitivity due to the light reflection. $75 \%$ of room coverage can achieve the PEs of less than $0.25 \mathrm{~m}$.

Fig. 13 (c) plots the PEs distribution of five ranges. According to the results, our system has a probability of $60.2 \%$ to achieve a PE less than $0.2 \mathrm{~m}$ and only $15.8 \%$ to have PEs in the range $0.3-0.4 \mathrm{~m}$. Thus, we can say that our proposal can guarantee a reasonable high-accuracy positioning ability.

\section{System Features AND CAPABILITIES}

The proposed system inherits the advantages of VLC-based positioning system and meanwhile overcomes some shortcomings in current positioning systems, such as the low-accuracy by using the radio beacon, high-cost of mmWave frontend and so on. The features and capabilities of this system in museum scenario can be summarized as follows.

\section{A. High-Accuracy}

This system provides a reasonable high positioning accuracy with a mean PEs of $0.18 \mathrm{~m}$ obtained by single-tone training signals. It allows visitors to match precisely their geographic location to the surrounding location-related information.

\section{B. High-Capacity}

The high-capacity can be illustrated in the following aspects: 1) a wide capacity is guaranteed by designing of LED's layout; 2) many spectrally efficient modulation formats cooperation with different digital signal processing techniques could provide the high-capacity. For example, wavelength division multiplexing (WDM) could support multiple users with different colors LED [49], space division multiple access (SDMA) could create parallel spatial pipes by differing spatial locations of the users and hence improve the capacity [50]. All these aspects enable users to seamlessly access the Internet while keeping high Quality of service (QoS) levels, avoiding network congestions and delays.

\section{Radiation-Free}

With LED Lamp, the system utilizes unlicensed visible light which has no radiation and no interference issues to broadcast geographic data. Based on this feature, the system and the broadcasting service can be applied more widely in indoor environment.

\section{Low-Cost}

The cost is lower because the VLC uses the existing lighting infrastructure to communicate and illuminate without additional installation, and the cost of the VLC front-end is only a LED lamp price with a small LED driver. 


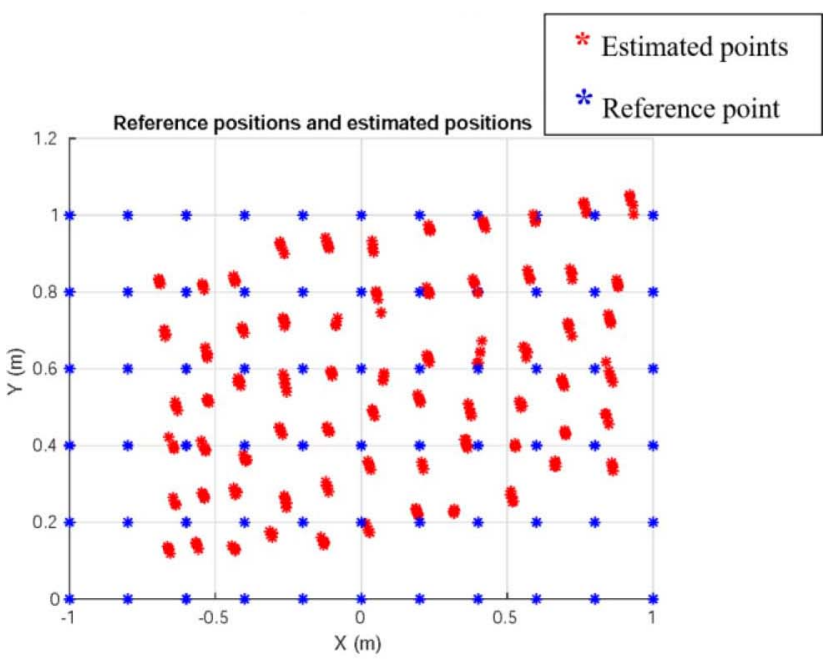

(a)

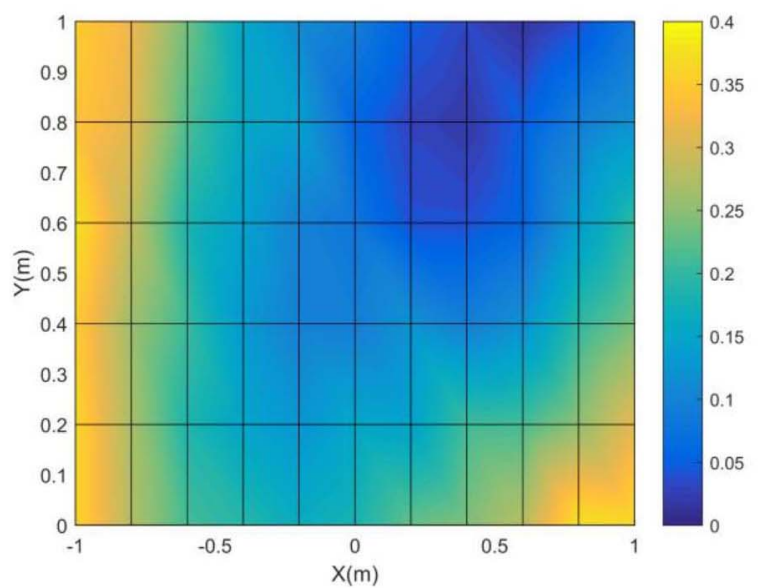

(b)

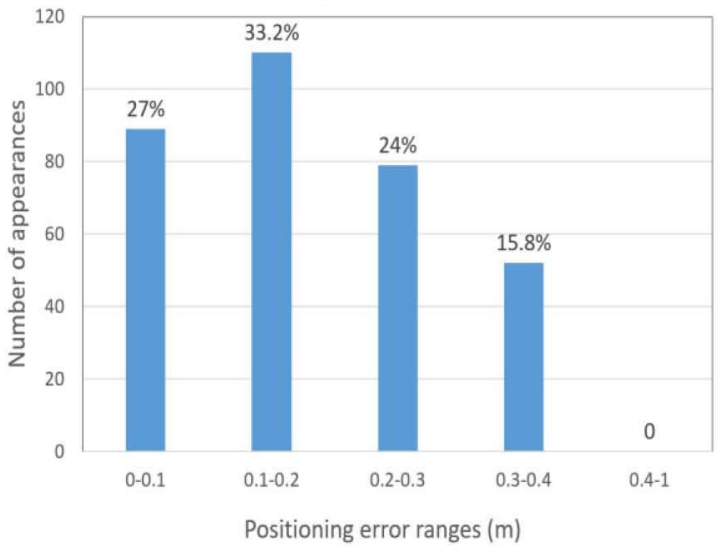

(c)

Fig. 13. (a) The distribution of reference points and estimated points. (b) The distribution of PEs. (c) The distribution of PEs among five error ranges.

\section{CONCLUSION}

In this paper, a 5G indoor positioning system for broadcasting service in the museum is proposed. This system uses LED devices for indoor positioning as well as indoor broadband communication, which is low-cost, radiation-free and easy to install. The definition of geographic data, the location-related data and a positioning procedure are proposed.
A demonstration with white LED lamps and commercial APD shows that the system has a high data rate of around 45.25 Mbps in the laboratory environment and a mean PEs of $0.18 \mathrm{~m}$. Besides that, the system has many other excellent features, such as high communication bandwidth, low-cost, radiation-free etc., so we do believe that the system can be widely used in the future in different indoor broadcasting service scenarios such as museums, shopping centers etc.

Moreover, based on these experimental results obtained in this paper, we summarized the key points of future work in order to improve system performance. First of all, as for the RSS-based VLC indoor positioning systems, many research works demonstrated that the calibration during offline preparation can improve the positioning accuracy [51]. Thus, our next work should focus on this and carefully analyze the effect on positioning accuracy and adopt appropriate calibration method in our system.

Secondly, the positioning errors have been measured to be relatively high on the edges, or corners, of the experimental environment. This may be caused by the multipath effect [12], [52]. Therefore, in order to boost the positioning accuracy, it is absolutely necessary to analyze the characteristics of practical indoor optical wireless channel in near future.

Thirdly, the current experimental results are based on RSS positioning algorithm and we will try to analyze and compare the performance of various positioning algorithms under the same experimental condition in our laboratory. These more comprehensive experimental results will be obtained and reported in the future.

Finally, to guarantee a high positioning accuracy, one technology will not be enough because of the complex indoor environment. It is necessary to propose an enhanced positioning system in the near future that fuses different positioning data to compensate for some inherent defect of visible light (such as impassable via opaque obstacles).

\section{REFERENCES}

[1] J. J. Gimenez et al., "5G new radio for terrestrial broadcast: A forwardlooking approach for NR-MBMS," IEEE Trans. Broadcast., vol. 65, no. 2, pp. 356-368, Jun. 2019.

[2] S. Ahn, K. Kim, S. Myung, S. Park, and K. Yang, "Comparison of lowdensity parity-check codes in ATSC 3.0 and 5G standards," IEEE Trans. Broadcast., vol. 65, no. 3, pp. 489-495, Sep. 2019.

[3] F. Alvarez et al., "An edge-to-cloud virtualized multimedia service platform for 5G networks," IEEE Trans. Broadcast., vol. 65, no. 2, pp. 369-380, Jun. 2019.

[4] S. Rommel, D. Perez-Galacho, J. M. Fabrega, R. Muñoz, S. Sales, and I. T. Monroy, "High-capacity 5G Fronthaul networks based on optical space division multiplexing," IEEE Trans. Broadcast., vol. 65, no. 2, pp. 434-443, Jun. 2019.

[5] NGMN 5G White Paper. Next Generation Mobile Networks, Frankfurt, Germany, NGMN Alliance, White Paper, 2015.

[6] N. Jawad et al., "Smart television services using NFV/SDN network management," IEEE Trans. Broadcast., vol. 65, no. 2, pp. 404-413, Jan. 2019.

[7] "Cisco visual networking index: Global mobile data traffic forecast update, 2017-2022," San Jose, CA, USA, Cisco, White Paper, 2019.

[8] Y. Jeong, S. Cho, G. Kim, C. Ahn, S. Lee, and W. Kim, "Location based service based on digital multimedia broadcasting," in IEEE Dig. Tech. Papers Int. Conf. Consum. Electron., Jan. 2006, pp. 147-148.

[9] A. Basiri et al., "Indoor location-based services challenges, requirements and usability of current solutions," Comput. Sci. Rev., vol. 24, pp. 1-12, May 2017. 
[10] IoRL. Accessed: Nov. 7, 2019. [Online]. Available: https://iorl.5gppp.eu/

[11] R. Abbas, K. Michael, and M. G. Michael, "Locationbased privacy, protection, safety, and security," in Privacy in a Digital, Networked World. Cham, Switzerland: Springer, 2015, pp. 391-414.

[12] S. Mazuelas, A. Conti, J. C. Allen, and M. Z. Win, "Soft range information for network localization," IEEE Trans. Signal Process., vol. 66, no. 12, pp. 3155-3168, Jun. 2018.

[13] S. Bartoletti, A. Giorgetti, M. Z. Win, and A. Conti, "Blind selection of representative observations for sensor radar networks," IEEE Trans. Veh. Technol., vol. 64, no. 4, pp. 1388-1400, Apr. 2015.

[14] A. Conti, D. Dardari, M. Guerra, L. Mucchi, and M. Z. Win, "Experimental characterization of diversity navigation," IEEE Syst. J., vol. 8, no. 1, pp. 115-124, Mar. 2014.

[15] A. Conti, M. Guerra, D. Dardari, N. Decarli, and M. Z. Win, "Network experimentation for cooperative localization," IEEE J. Sel. Areas Commun., vol. 30, no. 2, pp. 467-475, Feb. 2012.

[16] H. Wymeersch, J. Lien, and M. Z. Win, "Cooperative localization in wireless networks," Proc. IEEE, vol. 97, no. 2, pp. 427-450, 2009.

[17] B. Hanssens et al., "An indoor variance-based localization technique utilizing the UWB estimation of geometrical propagation parameters," IEEE Trans. Antennas Propag., vol. 66, no. 2, pp. 2522-2533, May 2018.

[18] S. Shrestha, J. Talvitie, and E. S. Lohan, "Deconvolution-based indoor localization with WLAN signals and unknown access point locations," in Proc. IEEE Int. Conf. Localization GNSS (ICL-GNSS), Jun. 2013, pp. 1-6.

[19] V. Moghtadaiee and A. G. Dempster, "Indoor location fingerprinting using FM radio signals," IEEE Trans. Broadcast., vol. 60, no. 2, pp. 336-346, Jun. 2014.

[20] R. Faragher and R. Harle, "Location fingerprinting with Bluetooth low energy beacons," IEEE J. Sel. Areas Commun., vol. 33, no. 11, pp. 2418-2428, Nov. 2015.

[21] A. Basiri, P. Peltola, P. F. e Silva, E. S. Lohan, T. Moore, and C. Hill, "Indoor positioning technology assessment using analytic hierarchy process for pedestrian navigation services," in Proc. Int. Conf. Location GNSS (ICL-GNSS), Jun. 2015, pp. 1-6.

[22] J. Luo, L. Fan, and H. Li, "Indoor positioning systems based on visible light communication: State of the art," IEEE Commun. Surveys Tuts., vol. 19, no. 4, pp. 2871-2893, 4th Quart., 2017.

[23] J. Saloranta and G. Destino, "On the utilization of MIMO-OFDM channel sparsity for accurate positioning," in Proc. 24th Eur. Signal Process. Conf. (EUSIPCO), Aug. 2016, pp. 748-752.

[24] Z. Lin, T. Lv, and P. T. Mathiopoulos, "3-D indoor positioning for millimeter-wave massive MIMO systems," IEEE Trans. Commun., vol. 66, no. 6, pp. 2472-2486, Jun. 2018.

[25] M. F. Keskin, A. D. Sezer, and S. Gezici, "Localization via visible light systems," Proc. IEEE, vol. 106, no. 6, pp. 1063-1088, 2018.

[26] Y. Almadaniet al., "A novel 3D visible light positioning method using received signal strength for industrial applications," Electronics, vol. 8, no. 2, p. 1311, 2019.

[27] S. Bartoletti, W. Dai, A. Conti, and M. Z. Win, "A mathematical model for wideband ranging," IEEE J. Sel. Topics Signal Process., vol. 9, no. 2, pp. 216-228, Mar. 2015.

[28] D. Plets, S. Bastiaens, L. Martens, W. Joseph, and N. Stevens, "On the impact of LED power uncertainty on the accuracy of $2 \mathrm{D}$ and 3D visible light positioning," Optik, vol. 195, Oct. 2019, Art. no. 163027.

[29] E. M. Jeong, S. H. Yang, H. S. Kim, and S. K. Han, "Tilted receiver angle error compensated indoor positioning system based on visible light communication," Electron. Lett., vol. 49, no. 14, pp. 890-892, 2013.

[30] P. Du, S. Zhang, C. Chen, A. Alphones, and W.-D. Zhong, "Demonstration of a low-complexity indoor visible light positioning system using an enhanced TDOA scheme," IEEE Photon. J., vol. 10, no. 4, pp. 1-10, Aug. 2018.

[31] M. S. Islim et al., "Towards $10 \mathrm{~Gb} / \mathrm{s}$ orthogonal frequency division multiplexing-based visible light communication using a GaN violet micro-LED," Photon. Res., vol. 5, no. 2, pp. A35-A43, 2017.
[32] G. Zhang, J. Zhang, X. Hong, and S. He, "Low-complexity frequency domain nonlinear compensation for OFDM based high-speed visible light communication systems with light emitting diodes," Opt. Exp., vol. 25, no. 4, pp. 3780-3794, 2017.

[33] T. Wu et al., "Tricolour R/G/B laser diode based eye-safe white lighting communication beyond 8 Gbit/s," Sci. Rep., vol. 7, p. 11, Jan. 2017.

[34] D. Tsonev, S. Videv, and H. Haas, "Towards a $100 \mathrm{~Gb} / \mathrm{s}$ visible light wireless access network," Opt. Exp., vol. 23, no. 2, pp. 1627-1637, 2014.

[35] D. Plets et al., "Three-dimensional visible light positioning: An experimental assessment of the importance of the LEDs' locations," in Proc. IEEE Int. Conf. Indoor Position. Indoor Navig. (IPIN), 2019, pp. 1-6.

[36] T. Q. Wang, Y. A. Sekercioglu, A. Neild, and J. Armstrong, "Position accuracy of time-of-arrival based ranging using visible light with application in indoor localization systems," J. Lightw. Technol., vol. 31 no. 20, pp. 3302-3308, Oct. 15, 2013.

[37] P. Du et al., "Experimental demonstration of 3D visible light positioning using received signal strength with low-complexity trilateration assisted by deep learning technique," IEEE Access, vol. 7, pp. 93986-93997, 2019.

[38] Y. Wu, X. Liu, W. Guan, B. Chen, X. Chen, and C. Xie, "High-speed 3D indoor localization system based on visible light communication using differential evolution algorithm," Opt. Commun., vol. 424, pp. 177-189, Oct. 2018.

[39] X. Y. Lin et al., "A mobile indoor positioning system based on iBeacon technology," in Proc. 37th Annu. Int. Conf. IEEE Eng. Med. Biol. Soc. (EMBC), Aug. 2015, pp. 4970-4973.

[40] A. Handojo, R. Lim, T. Octavia, and J. K. Anggita, "Museum interactive information broadcasting using indoor positioning system and Bluetooth low energy: A pilot project on Trowulan museum Indonesia," in Proc. 3rd Technol. Innov. Manag. Eng. Sci. Int. Conf. (TIMES-iCON), 2008, pp. $1-5$.

[41] A. Kuusik, S. Roche, and F. Weis, "SmartMuseum: Cultural content recommendation system for mobile users," in Proc. IEEE 4th Int. Conf. Comput. Sci. Converg. Inf. Technol., 2009, pp. 477-482.

[42] J. L. Lugrin et al., "A location-based VR museum," in Proc. 10th Int. Conf. Virtual Worlds Games Serious Appl. (VS-Games), Sep. 2018, pp. $1-2$.

[43] S. Bastiaens, W. Raes, N. Stevens, W. Joseph, and D. Plets, "New photodiode responsively model for RSS-based VLP," in Proc. Global LIFI Congr. (GLC), Jun. 2019, pp. 1-6.

[44] H. Steendam, "A 3-D positioning algorithm for AOA-based VLP with an aperture-based receiver," IEEE J. Sel. Areas Commun., vol. 36. no. 1, pp. 23-33, Jan. 2018

[45] S. Y. Jung, S. Hann, S. Park, and C. S. Park, "Optical wireless indoor positioning system using light emitting diode ceiling lights," Microw. Opt. Technol. Lett., vol. 54, no. 7, pp. 1622-1626, 2012.

[46] E. Woods et al., "Augmenting the science centre and museum experience," in Proc. ACM 2nd Int. Conf. Comput. Graph. Interactive Techn. Australasia South East Asia, Jun. 2004, pp. 230-236.

[47] L. Shi, W. Li, X. Zhang, Y. Zhang, G. Chen, and A. Vladimirescu, "Experimental 5G new radio Integration with VLC," in Proc. 25th IEEE Int. Conf. Electron. Circuits Syst. (ICECS), Dec. 2018, pp. 61-64.

[48] Evolved Universal Terrestrial Radio Access (E-UTRA); Base Station (BS) Radio Transmission and Reception, V14, 3GPP Standard TS 36.104, 2009.

[49] F.-M. Wu, C.-T. Lin, C.-C. Wei, C.-W. Chen, Z.-Y. Chen, and H.-T. Huang, "3.22-Gb/s WDM visible light communication of a single RGB LED employing carrier-less amplitude and phase modulation," in Proc. IEEE Opt. Fiber Commun. Conf. Exposit. Nat. Fiber Optic Eng. Conf. (OFC/NFOEC), Mar. 2013, pp. 1-3.

[50] L. Yin, $\mathrm{X}$. Wu, and $\mathrm{H}$, Haas, "SDMA grouping in coordinated multi-point VLC systems," in Proc. IEEE Summer Topicals Meeting Series (SUM), Jul. 2015, pp. 169-170.

[51] R. Amsters et al., "Towards automated calibration of visible light positioning systems," in Proc. Int. Conf. Indoor Position. Indoor Navig. (IPIN), 2019, pp. 1-8.

[52] A. Conti, S. Mazuelas, S. Bartoletti, W. C. Lindsey, and M. Z. Win, "Soft information for localization-of-things," Proc. IEEE, vol. 107, no. 11, pp. 2240-2264, 2019. 